\title{
Neuromuscular correlates of subthalamic stimulation and upper limb freezing in Parkinson's disease
}

\author{
Marlieke Scholten $^{\text {a,b,c,d,*, Rosa Klotz }}{ }^{\text {a,b,c }}$, Christian Plewnia ${ }^{\text {c,f }}$, Tobias Wächter ${ }^{\mathrm{e}}$, Carina Mielke ${ }^{\mathrm{a}, \mathrm{b}, \mathrm{c}}$, \\ Bastiaan R. Bloem ${ }^{\mathrm{g}}$, Christoph Braun ${ }^{\mathrm{c}, \mathrm{h}, \mathrm{i}}$, Ulf Ziemann ${ }^{\mathrm{c}, \mathrm{j}}$, Rathinaswamy B. Govindan ${ }^{\mathrm{k}}$, \\ Alireza Gharabaghi ${ }^{c, 1}$, Rejko Krüger ${ }^{a, b, c, m}$, Daniel Weiss ${ }^{\text {a,b,c,* }}$
}

${ }^{a}$ Department of Neurodegenerative Diseases and Hertie Institute for Clinical Brain Research (HIH), University of Tuebingen, Germany

${ }^{\mathrm{b}}$ German Centre of Neurodegenerative Diseases (DZNE), University of Tuebingen, Tuebingen, Germany

${ }^{\mathrm{c}}$ Centre for Integrative Neuroscience (CIN), University of Tuebingen, Tuebingen, Germany

${ }^{\mathrm{d}}$ Graduate School of Neural \& Behavioural Sciences, International Max Planck Research School, University of Tuebingen, Tuebingen, Germany

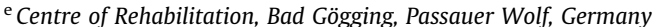

${ }^{\mathrm{f}}$ Department of Psychiatry and Psychotherapy, University of Tuebingen, Tuebingen, Germany

${ }^{\mathrm{g}}$ Radboud University Medical Centre, Donders Institute for Brain, Cognition and Behavior, Department of Neurology, Nijmegen, The Netherlands

${ }^{\mathrm{h}}$ MEG Center, University of Tuebingen, Tuebingen, Germany

${ }^{\mathrm{i}} \mathrm{CIMeC}$, Center of Mind/Brain Sciences, University of Trento, Italy

j Department of Neurology and Stroke, and Hertie Institute for Clinical Brain Research (HIH), University of Tuebingen, Tuebingen, Germany

${ }^{\mathrm{k}}$ Division of Fetal and Transitional Medicine, Children's National Medical Center, Washington, DC, USA

${ }^{1}$ Division of Functional and Restorative Neurosurgery, Department of Neurosurgery, University of Tuebingen, Tuebingen, Germany

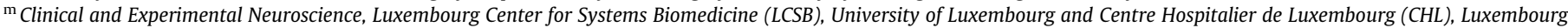

\section{A R T I C L E I N F O}

\section{Article history}

Accepted 13 February 2015

Available online 28 February 2015

\section{Keywords:}

Parkinson's disease

EEG

Subthalamic nucleus

Deep brain stimulation

Upper limb freezing

Freezing of gait

\section{H I G H L I G H T S}

- Subthalamic stimulation reduces exaggerated low-frequency intermuscular coherence during continuous fingertapping in iPD.

- We implemented criteria to detect and segment freezing events of upper limb movement for electrophysiological data analyses.

- Freezing of upper limb movement presents with increased low-frequency muscular and cortical activation, likely reflecting increased cortical motor inhibition.

\section{A B S T R A C T}

Objective: The pathophysiology of deep brain stimulation mechanisms and resistant freezing phenomena in idiopathic Parkinson's disease (iPD) remains incompletely understood. Further studies on the neuromuscular substrates are needed.

Methods: We analyzed 16 patients with advanced iPD and bilateral subthalamic nucleus stimulation, and 13 age- and gender-matched healthy controls. Patients were tested after overnight withdrawal of medication with 'stimulation off (StimOff) and 'stimulation on' (StimOn). Subjects performed continuous tapping of the right index finger with simultaneous recordings of biomechanical registration, EMG of finger flexors and extensors, and EEG. First, we analyzed EEG and EMG spectral measures comparing StimOff with healthy controls and StimOff with StimOn (irrespective of freezing). Second, we contrasted 'regular (unimpaired) tapping' and 'freezing' resistant to subthalamic neurostimulation as obtained in StimOn.

Results: iPD showed increased intermuscular coherence around $8 \mathrm{~Hz}$ in StimOff that was reduced in StimOn. This $8 \mathrm{~Hz}$ muscular activity was not coherent to cortical activity. 'Freezing' episodes showed increased muscle activity of finger flexors and extensors at $6-9 \mathrm{~Hz}$, and increased cortical activity at

\footnotetext{
Abbreviations: ED, extensor digitorum communis muscle; EEG, electroencephalography; EMG, electromyography; FD, flexor digitorum superficialis muscle; iPD,

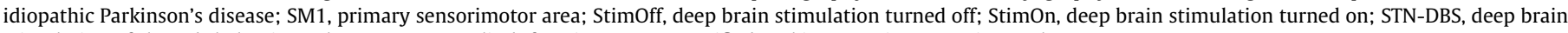
stimulation of the subthalamic nucleus; ULF, upper limb freezing; UPDRS, Unified Parkinson's Disease Rating Scale.

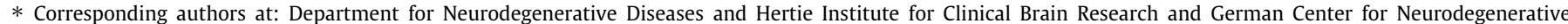
Diseases, University of Tuebingen, Hoppe-Seyler-Str. 3, 72076 Tuebingen, Germany. Tel.: +49 70712985650 ; fax: +49 7071 298349.

E-mail addresses: marlieke.scholten@uni-tuebingen.de (M. Scholten),daniel.weiss@uni-tuebingen.de (D. Weiss).
} 
7-11 Hz. During transition from regular tapping to 'freezing' the cortical activity first increased over the left sensorimotor area followed by a spread to the left frontal and right parietal areas.

Conclusions: We identified neuromuscular motor network features of subthalamic neurostimulation therapy and resistant upper limb freezing that point to increased low-frequency muscular and cortical activity.

Significance: Together, our findings demonstrate several motor network abnormalities associated with upper limb freezing that may translate into future research on freezing of gait in iPD.

(C) 2015 International Federation of Clinical Neurophysiology. Published by Elsevier Ireland Ltd. All rights reserved.

\section{Introduction}

Symptomatic treatment of idiopathic Parkinson's disease (iPD) includes deep brain stimulation of the subthalamic nucleus (STNDBS) to control segmental motor symptoms and motor fluctuations (Deuschl et al., 2006; Schuepbach et al., 2013). However, freezing shows a less favorable therapeutic response (Vercruysse et al., 2014; Weiss et al., 2013) and a closer pathophysiological understanding is highly warranted. Functional imaging pointed to both cortical and subcortical network abnormalities in freezers, and implicated the subthalamic nucleus, as well as the mesencephalic locomotor region and higher-order cortical areas in freezing pathophysiology (Herman et al., 2013; Shine et al., 2013b). Much less is known about the electrophysiological neuromuscular mechanisms behind freezing (Nutt et al., 2011).

Here, we studied the motor network mechanisms of two major clinical issues: first, we explored the effect of subthalamic neurostimulation on the motor network characteristics during fingertapping (irrespective of freezing). This is of interest, as several studies pointed to large-scale motor network effects of DBS. As such, subthalamic neurostimulation modulated cortical activity in terms of movement-related desynchronization (Devos et al., 2004; Weiss et al., 2015). Moreover, STN-DBS increased motor cortex excitability (Kuriakose et al., 2010). Similarly, there is evidence for both defective corticospinal interactions and muscle activation at abnormally low frequencies $<10 \mathrm{~Hz}$ in the clinical 'off state (Salenius et al., 2002; Weiss et al., 2012). As spinal antagonistic motor neurons have segregated motor neuron pools (Jessell et al., 2011), antagonistic motor control can be studied with surface EMG. A distinct impairment of antagonistic spinal reciprocal inhibition via inhibitory 1a-interneurons was demonstrated (Meunier et al., 2000). Moreover, both cortical (Bertolasi et al., 1998) and subcortical modulatory influences (Raoul et al., 2012) on antagonistic motor control were revealed.

Second, we captured freezing events of continuous fingertapping (referred to as 'upper-limb freezing', ULF) and compared freezing to regular movement. Freezing phenomena are not restricted to gait, but may entrain fingertapping as well. ULF and freezing of gait occur with high clinical co-incidence (Nieuwboer et al., 2009; Vercruysse et al., 2013), although there is an ongoing controversy whether both phenomena share common network mechanisms (Barbe et al., 2014). As common characteristic, both ULF and freezing of gait showed increased motor output in the frequency range from 4 to $8 \mathrm{~Hz}$, whereas regular fingertapping occurred from 1 to $3 \mathrm{~Hz}$ (Vercruysse et al., 2012). Previous functional imaging research pointed to a specific role of the subthalamic nucleus in gait freezers (Fling et al., 2013; Shine et al., 2013a; Vercruysse et al., 2013). Similar evidence came from clinical studies that pointed to basal ganglia outputs in terms of STN (Vercruysse et al., 2014) and the substantia nigra pars reticulata (Weiss et al., 2013) as important modulators of gait freezing. Moreover, cortical processing as well as subcortico-cortical interactions seem to be relevant. In this sense, patients with gait freezing showed higher functional connectivity between the supplementary motor area and the mesencephalic locomotor region (Fling et al., 2013). Moreover, ULF was paralleled by increased cortical BOLD activity in the left dorsolateral prefrontal cortex, motor cortex, supplementary motor area, and dorsal premotor area (Vercruysse et al., 2013). A recent EEG study found increased cortical theta band activity during both transition from normal movement to freezing and freezing itself (Shine et al., 2014). Together, these findings substantiate the view that freezing phenomena are paralleled by maladaptive processing on both cortical and subcortical levels.

We recorded iPD patients with STN-DBS and healthy controls with biomechanical registration, EEG, and EMG of antagonistic muscles during continuous fingertapping. We studied the electrophysiological neuromuscular mechanisms, i.e., (i) activation patterns of cortex and antagonistic muscles, (ii) synchronization between antagonistic muscles (intermuscular coherence), and (iii) synchronization between cortex and muscles (corticomuscular coherence) on two major clinical issues. First, we studied iPD patients both in 'stimulation off' (StimOff) and 'stimulation on' (StimOn) conditions, and compared StimOff to age- and gendermatched healthy controls. We hypothesized that iPD show increased intermuscular coherence in StimOff, and we postulated that subthalamic stimulation would decrease it. Second, we introduced additional dual task interference in the StimOn condition in order to provoke freezing phenomena, i.e., ULF (Spildooren et al., 2010). We selected StimOn in order to capture freezing episodes resistant to STN-DBS therapy. We hypothesized that ULF shows increased cortical activity since iPD motor impairment demonstrated with impaired cortical activity desynchronization (Devos et al., 2004; Weiss et al., 2015), and since inhibition of preplanned movement was associated with transient cortical activity increase (Sauseng et al., 2012). Moreover, we expected increased muscular low-frequency activation and increased intermuscular coherence during freezing, the latter as a consequence of impaired antagonistic motor control.

\section{Methods}

\subsection{Subject characteristics}

Seventeen iPD patients treated with STN-DBS and fourteen ageand gender matched healthy controls (HC) were recruited from the Department for Neurodegenerative Diseases, University of Tuebingen, Germany. Two subjects were excluded from the analyses (one iPD patient did not tolerate the discontinuation of the DBS therapy, and one HC did not adhere to the task requirements). Therefore, data are presented from sixteen iPD patients (iPD1iPD16, eleven male, age $61.2 \pm 11.1$ years) and thirteen age- and gender-matched $\mathrm{HC}$ (HC1-HC13, eight male, age $62.6 \pm 10.6$ years). 
The disease duration of iPD patients was $13.6 \pm 6.8$ years. Inclusion criteria were: patients diagnosed with iPD and akinetic-rigid symptom dominance (determined by neurologist and patient history), treatment with STN-DBS for at least 3 months, and age $>18$ years. The akinetic-rigid symptom dominance was further supported as patients were examined in 'stimulation off (StimOff) during the study (UPDRS III: item 20 'tremor' of head, upper- and lower limbs summed; median [IQR] 0.0 [0.0 1.5]; item 22 'rigidity' of head and upper limbs summed: 5.5 [5.0 8.0]; items 23-26 'diadochokinesia': 20.0 [14.8 23.3]). Exclusion criteria were: resting tremor, cognitive impairment (Mini Mental State Examination <25 (Folstein et al., 1975)) and other competing neurological or neuromuscular disease conditions. The study was approved by the local Ethics committee of the University of Tuebingen in accordance to the Declaration of Helsinki. All subjects provided written informed consent prior to study inclusion. All subjects were right-handed as documented with the Edinburgh Handedness Scale. All patients were identified as 'freezers' (score $>1$ ) according to the Freezing of Gait Questionnaire (FOGQ; Giladi et al., 2000), however gait 'freezing' was not treated as formal inclusion criterion. The iPD patient characteristics are displayed in Table 1.

\subsection{Experimental set up and paradigm}

Anamnestic scores (FOG-Q, MMST, and Edinburgh handedness score) were determined on the day before the recordings. All recordings took place in the morning after overnight withdrawal of dopaminergic medication. UPDRS III was determined in both StimOff and StimOn. Subjects were seated comfortably with the right arm supported by an arm rest. They were instructed to perform continuous tapping with the right index finger as quickly and precisely as possible, whilst the index finger was in permanent contact with the force transducer. The subjects were instructed to precisely push the force transducer with a predefined strength of $2 \mathrm{~N}$, and were provided with ongoing visual real-time feedback of the exerted pressure on a computer-screen placed $1 \mathrm{~m}$ in front. Due to the weak contraction strength and the permanent contact of finger and force transducer, the performance of this motor task required only minimum spatial displacement of a few millimeters of the index finger when exerting the pressure. This experimental setup was chosen to stabilize the tapping and movement characteristics across patients and subjects. Since modulation and rescaling of the contraction strength are known to modulate the magnitude of corticomuscular coherence, we aimed to standardize the contraction strength by visual feedback (Kilner et al., 2000; Mima and Hallett, 1999). Importantly, no external cues or rhythms were presented that could have prevented freezing episodes (Giladi and Hausdorff, 2006; Rahman et al., 2008). All subjects and patients were instructed to tap continuously and - if present - to try immediately to overcome movement arrests. Subjects performed continuous fingertapping in ten blocks of $20 \mathrm{~s}$. Between blocks a pause of ten seconds was given to prevent fatigue.

\subsection{Electrophysiological recordings}

During the fingertapping, bipolar surface EMG of the flexor digitorum superficialis (FD) and extensor digitorum communis (ED) muscles using $\mathrm{Ag} / \mathrm{AgCl}$ electrodes (Myotronics, Kent, USA) and a 36-channel EEG were recorded simultaneously to the biomechanical signal measured by the force transducer. For the EEG-recordings, linked earlobes were used as reference and FPz was used as ground electrode. All signals were sampled at $1000 \mathrm{~Hz}$ and monitored online using a BrainVision Recorder (Brain Products version 1.20, MES Electronics, Gilching, Germany). Impedances were kept below $5 \mathrm{kOhm}$.

\subsection{Differences between HC and iPD StimOff, and effect of subthalamic stimulation}

First, HC and iPD patients were recorded with 'fingertapping only'. We aimed to compare iPD patients in the StimOff condition with HC. Next, we studied the influence of STN-DBS and compared the StimOff and StimOn conditions. Therefore, the iPD patients performed 'fingertapping only' in both StimOff and StimOn in randomized order. In order to limit the potential influence of clinical carry-over effects, each treatment condition was active for at least 20 min prior to the recordings. This is considered sufficient to limit potential carry-over effects in advanced disease stages (Cooper et al., 2013). Bipolar stimulation settings were used in StimOn to minimize DBS artifacts in the EEG recordings. If pre-existing, the best individual bipolar parameters were maintained. In case of

Table 1

Baseline characteristics of the iPD patients.

\begin{tabular}{|c|c|c|c|c|c|c|c|c|}
\hline \multirow[t]{2}{*}{ Number } & \multirow[t]{2}{*}{ Age (yrs) } & \multirow[t]{2}{*}{ Gender } & \multirow[t]{2}{*}{ Disease duration (yrs) } & \multirow[t]{2}{*}{ FOG-Q } & \multicolumn{2}{|c|}{ UPDRS III ('18-26’) } & \multicolumn{2}{|c|}{ 'Diadochokinesia' } \\
\hline & & & & & StimOff & StimOn & StimOff & StimOn \\
\hline $1^{*}$ & 61 & M & 7 & 2 & 18 & 11 & 10 & 7 \\
\hline $2^{*}$ & 53 & M & 10 & 5 & 56 & 39 & 30 & 20 \\
\hline 3 & 58 & M & 15 & 2 & 22 & 14 & 7 & 2 \\
\hline 4 & 74 & M & 8 & 7 & 32 & 12 & 17 & 9 \\
\hline 5 & 67 & M & 25 & 10 & 25 & 18 & 16 & 11 \\
\hline $6^{*}$ & 41 & M & 10 & 10 & 16 & 8 & 9 & 4 \\
\hline 7 & 63 & M & 14 & 3 & 27 & 19 & 19 & 12 \\
\hline 8 & 67 & $\mathrm{~F}$ & 16 & 15 & 36 & 33 & 24 & 21 \\
\hline $9^{*}$ & 65 & $\mathrm{~F}$ & 24 & 15 & 29 & 22 & 15 & 12 \\
\hline 10 & 68 & $\mathrm{~F}$ & 15 & 13 & 24 & 15 & 14 & 10 \\
\hline 11 & 64 & $\mathrm{~F}$ & 12 & 15 & 31 & 27 & 22 & 20 \\
\hline $12^{*}$ & 63 & $\mathrm{~F}$ & 19 & 14 & 32 & 25 & 21 & 20 \\
\hline $13^{*}$ & 32 & $\mathrm{M}$ & 2 & 6 & 42 & 30 & 28 & 20 \\
\hline $14^{*}$ & 74 & $\mathrm{M}$ & 8 & 8 & 34 & 25 & 23 & 18 \\
\hline $15^{*}$ & 66 & $\mathrm{M}$ & 8 & 4 & 35 & 23 & 22 & 19 \\
\hline $16^{*}$ & 63 & $\mathrm{M}$ & 24 & 15 & 45 & 34 & 28 & 21 \\
\hline
\end{tabular}

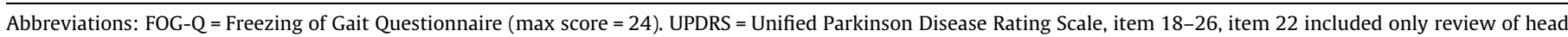

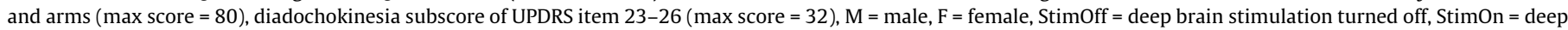
brain stimulation turned on.

* Patients with freezing of upper limb. 
chronic treatment with monopolar stimulation, a bipolar program similar to the effective monopolar setting was established: therefore, we held the negative active contact constant and polarized against the most dorsal neighboring contact. In this situation, amplitudes were increased by $30 \%$ in order to achieve similar clinical efficacy (Silberstein et al., 2005). All patients received high frequency stimulation (twelve patients at $130 \mathrm{~Hz}$, three at $125 \mathrm{~Hz}$, and one at $180 \mathrm{~Hz}$ ). The mean stimulation amplitudes were $3.7 \pm 1.0 \mathrm{~V}$ (left STN) and $3.4 \pm 1.1 \mathrm{~V}$ (right STN), and the mean pulse widths were $73.1 \pm 18.9 \mu$ s (left STN) and $67.5 \pm 13.4 \mu \mathrm{s}$ (right STN). The clinical motor state in StimOff and StimOn was documented (UPDRS III). Ratings were restricted to the UPDRS III items 18-26, and 'rigidity' item 22 was obtained from head and upper extremities only, as subjects were largely immobile in sitting positions owing to EEG and EMG installed. A 'diadochokinesia' subscore was summed from the UPDRS items 23-26.

\subsection{Comparison of 'regular tapping' and 'upper limb freezing'}

After the recordings with 'fingertapping only', iPD patients performed the fingertapping in StimOn during dual task interference (StimOn interference). We introduced dual task interference, in order to reliably obtain freezing episodes under research laboratory conditions (Camicioli et al., 1998; O'Shea et al., 2002; Spildooren et al., 2010). StimOn was considered as therapeutic condition, as we aimed to capture ULF resistant to subthalamic stimulation therapy as a widely recognized limitation of PD state-of-the-art therapy in advanced disease stages (Krack et al., 2003; Vercruysse et al., 2014; Weiss et al., 2013). We only analyzed the ULF episodes during StimOn interference in order to study freezing resistant to subthalamic stimulation. Although ULF episodes also occurred 'spontaneously' in StimOff (23 episodes in six patients) and to lesser extent in StimOn (five episodes in three patients), we did not concatenate ULF episodes across different clinical conditions. In the StimOn interference condition, we obtained clear-cut transitions from 'regular tapping' to 'freezing' (which is important for the time-frequency analysis as explained below) (Fig. 1). HC also performed the fingertapping during dual task interference. We chose a phonemic verbal fluency task in which the subjects generated as many words as possible from a given initial letter. All subjects received the same set of letters that consisted of the ten most-used German consonants (one consonant per block of $20 \mathrm{~s}$ ). The order of the presented letters was randomized across subjects. The subjects were instructed to equally share their attention between the index fingertapping and the verbal fluency task (Bloem et al., 2006). The performance on the verbal fluency task (number of words generated) was audio-recorded and evaluated offline. All subjects could generate words at each letter. The total number of words generated was $53.2 \pm 14.2$ in $\mathrm{HC}$ and $52.9 \pm 15.3$ in iPD patients. A one-way ANOVA was conducted to examine the difference between iPD patients and $\mathrm{HC}$ on the total number of words generated treating the years of education as covariate. Hence, there was no difference between HC and iPD in number of words generated $\left(F_{1,24}=0.445 ; P=0.511\right)$.

\subsection{Analyses}

Motor performance was analyzed as 'tapping frequency' and 'tapping regularity'. The tapping frequency was expressed as number of peaks over time $(\mathrm{Hz})$. The regularity of tapping was defined as coefficient of the peak-to-peak interval variability and given as the standard deviation of the interpeak interval normalized by the mean interpeak interval. EEG, EMG, and force transducer signals were analyzed with Matlab (R2012b, The Mathworks, Nattick, MA, USA) based on the open-source toolboxes EEGlab (v13.1.1b) (Delorme and Makeig, 2004) and Fieldtrip (Oostenveld et al., 2011).

For offline preprocessing (prior to any spectral analyses), EMG signals were band-pass filtered from 10 to $300 \mathrm{~Hz}$, notch filtered for the $50 \mathrm{~Hz}$ line artifact, and full-wave rectified. EMG can be considered as a broad-band oscillatory activity (carrier activity) whose amplitude is modulated by a low frequency signal $(<25 \mathrm{~Hz})$ (Farmer et al., 1993). In the spectrum, such modulation signals result in the broadening of the carrier band, yet, are not reflected by an individual peak in the low frequency range. Rectifying the band-pass filtered EMG signal is a simple but efficient approach to demodulate the EMG activity and to extract the low-frequency modulation signal (Ward et al., 2013). EEG signals were filtered from 1 to $200 \mathrm{~Hz}$ and notch filtered at $50 \mathrm{~Hz}$. For both EMG and EEG signals, a finite impulse response filter was used. EEG signals were visually inspected and a block of $20 \mathrm{~s}$ was rejected if it was affected by muscle artifacts. Similarly, time series were rejected if affected by tremor. EEG Principal Component Analysis was performed. Components were rejected if corresponding to eye blinks or to transient muscle artifacts (from facial or temporal muscles). Then, the remaining components were transformed back to the time domain. Reference-free EEG data were obtained using the Hjorth transform as spatial filter (Hjorth, 1980). From each of the performance blocks, the first two seconds and the last second were removed in order to achieve blocks with stable motor performance.

\subsubsection{Operationalized offline detection of upper limb freezing (ULF) episodes}

ULF episodes were selected and segmented semi-automatically to ensure reproducibility of our offline data-analyses. For this purpose, we used an operationalized algorithm that was recently developed to evaluate the clinical and biomechanical characteristics of freezing in upper limb movement (Nieuwboer et al., 2009). A time-series in the biomechanical signal was categorized as ULF episode, if (i) the amplitude deflection of the finger tap decreased below $1 \mathrm{~N}$ (i.e., below $50 \%$ of the requested force modulation of $2 \mathrm{~N}$ ), (ii) the duration exceeded one second, and (iii) the frequency of motor output increased above $3 \mathrm{~Hz}$ based on the biomechanical registration. (Fig. 1). We automatically selected all episodes satisfying criteria one and two, i.e., 50\% amplitude decrease lasting more than one second. Then, we visually determined if there was motor output at frequencies $>3 \mathrm{~Hz}$. The data length of 'regular tapping' was adjusted to the data length of ULF and balanced for the contribution of a single subject to the total data pool.

\subsubsection{Frequency domain analyses of cortical and muscular activity}

For 'fingertapping only', cortical and muscular activity and corticomuscular coherence were analyzed in the frequency domain using spectral analyses. EEG and EMG signals were divided into half-overlapping segments with a duration of two seconds. The choice of two seconds was made in order to achieve a frequency resolution of $0.5 \mathrm{~Hz}$. For data in each window, power (the squared absolute of the Fourier transform) and cross-spectrum, which is the product of the Fourier transform of one signal and the complex conjugate of the Fourier transform of the other signal, were calculated. These spectral quantities were averaged over all windows.

Coherence of the signals $x$ and $y$ is defined as the ratio of the estimate of the cross-spectrum, $P_{x y}$ at frequency $f$, to the product of the autospectra, $P_{x x}$ and $P_{y y}$ at frequency $f$ :

$\operatorname{Coh}_{x y}(f)=\frac{P_{x y}(f)^{2}}{P_{x x}(f) P_{y y}(f)}$ 

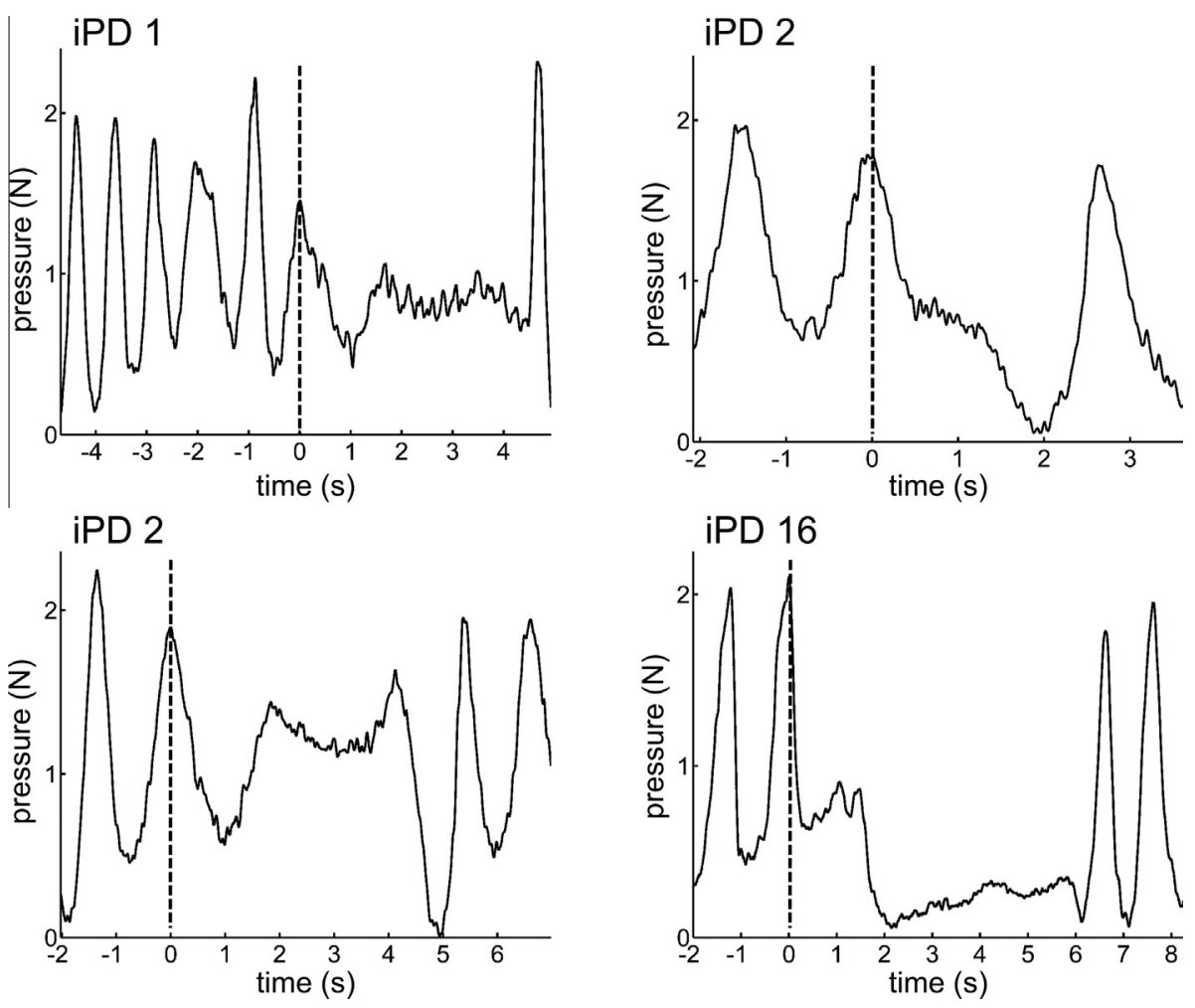

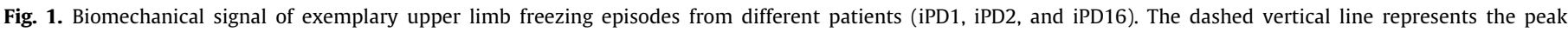
maximum of the last regular tap before freezing at time-point ' 0 '. $X$-axis: time (s); $Y$-axis: pressure force $(\mathrm{N})$.

Coherence is a normalized measure and takes on a value of one if there is an ideal correlation between the two signals and takes on a value of zero if there is no correlation between the two signals (Mima and Hallett, 1999). We calculated the intermuscular coherence between EMG signals in FD and ED, and the corticomuscular coherence between all EEG channels and EMG of FD and ED, respectively. Significance of the coherence estimates was decided on the 95\% confidence limit (Rosenberg et al., 1989).

For 'freezing' vs. 'regular tapping', muscular and cortical activity were analyzed for each ULF trial. Regular tapping trials were adjusted to the length of the ULF trials. Here, the power was calculated with a window-length of one second (because some trials were shorter than two seconds) and the window moved forward in steps of $0.1 \mathrm{~s}$, achieving a frequency resolution of $1 \mathrm{~Hz}$. The spectra were averaged over all windows and over all trials of the subject.

For both 'fingertapping only' and freezing vs. regular tapping both muscular and cortical activity are expressed as relative power by dividing the power amplitude at a distinct frequency bin by the mean power of the whole spectrum $(1-100 \mathrm{~Hz})$.

\subsubsection{Event-locked time-frequency analysis}

To characterize the cortical processing when regular movement turned into an ULF episode, time-frequency analysis was performed. Time-point ' 0 ' was set as the peak maximum of the last regular tap of the biomechanical signal before entering the ULF episode. We segmented the data from -1000 to $+2000 \mathrm{~ms}$ relative to time point ' 0 '. For each epoch, EEG signals were decomposed using a complex Morlet wavelet for frequencies from 3 to $25 \mathrm{~Hz}$ with a resolution of $1 \mathrm{~Hz}$ (Schneider et al., 2008). The whole epoch was normalized to account for the variability between the ULF episodes. This normalization is done by subtracting from all power values the time-averaged power at a distinct frequency bin, and subsequently by dividing all power values by the time-averaged standard deviation of the epoch (Roach and Mathalon, 2008).

\subsection{Statistical analyses}

Descriptive findings are reported as mean \pm standard deviation when tested with parametric tests and as median [interquartile range] in case of non-parametrical test procedures. We applied one-sided parametric tests for clinical effects and motor performance. Two-sided non-parametric tests were applied for spectral measures as they were mostly not normally distributed. First, we studied the differences between iPD in StimOff and HC using independent samples $t$-tests or a Mann-Whitney U tests. Next, we studied the influence of subthalamic stimulation by comparing StimOff vs. StimOn in iPD with paired samples $t$-tests or Wilcoxon tests.

'Freezing' was compared with 'regular tapping' (both selected from StimOn interference) using the paired samples $t$-test or Wilcoxon test. Correlation between the total duration of the ULF in each subject and question 3 of the FOG-Q was calculated using the non-parametric Spearman-Rho test. Cortical power, corticomuscular coherence, and time-frequency spectra were tested using a non-parametric cluster-based permutation test (Maris and Oostenveld, 2007). This approach addresses the need to correct for multiple comparisons by building clusters without losing sensitivity of spectral changes. First, a $t$-value is calculated for each sample with the predefined test-statistic (for example an independent samples $t$-test). Then the samples with $t$-values higher than threshold are clustered based on spatial adjacency (and additionally temporal adjacency in time-frequency series). For each cluster the sum of the $t$-values is taken. Then the significance probability is calculated by the Monte Carlo method, which approximated the permutation distribution of the test-statistic (maximum of the cluster-level summed $t$-values) by drawing 5000 random partitions. The Monte-Carlo significance probability was considered significant for two-tailed $P<0.05$.

Time-frequency spectra were analyzed for statistical significance by comparing distinct time intervals in the time range from 
0.5 to $1.5 \mathrm{~s}$ after the last regular finger tap with the time period one second before the last regular finger tap ( -1 to $0 \mathrm{~s}$ ) using the cluster-based permutation test explained above. For all tests, $P$ values $<0.05$ were considered significant. Statistics were performed using IBM SPSS statistics, version 22 (IBM Deutschland GmbH, Ehningen, Germany) and the Fieldtrip toolbox (Oostenveld et al., 2011).

\section{Results}

\subsection{Differences between iPD StimOff and HC, and effects of subthalamic stimulation}

\subsubsection{Finger tapping performance and clinical outcome}

During 'fingertapping only', stimulation improved bradykinesia according to the diadochokinesia UPDRS III subscore (Table 1 , StimOff $19.1 \pm 7.0$; StimOn $\left.14.1 \pm 6.5 ; t_{15}=7.93, P<0.001\right)$. The tapping frequency was slower in iPD StimOff compared to HC. StimOn increased the tapping frequency (Table 2).

\subsubsection{Antagonistic muscular activity, cortical activity and} corticomuscular coherence

Muscular activity spectra showed distinct peaks both at tapping frequency and at $8 \mathrm{~Hz}$ (Fig. 2). For statistical comparison, we used the individual tapping frequencies, and 'activity at $8 \mathrm{~Hz}$ ' was built as mean value from 7.5 to $8.5 \mathrm{~Hz}$.

At individual tapping frequency, agonistic FD muscle activity was similar in HC, iPD StimOff and StimOn (Table 2, Fig. 2A). The antagonistic ED muscle showed decreased activity in iPD StimOff compared with $\mathrm{HC}(P=0.012$, Fig. $2 \mathrm{~B})$, and this was unchanged in the StimOn condition (Fig. 2E). Intermuscular coherence showed neither a difference between StimOff and HC, nor between StimOff and StimOn.

Corticomuscular coherence over the left sensorimotor area (SM1) was taken at the individual tapping frequency as prominent coherence peak from either the $\mathrm{C} 3, \mathrm{C} 1, \mathrm{CP} 1$, or CP3 electrode. Corticomuscular coherence exceeded the 95\% confidence limit with both FD and ED in HC, StimOff, and StimOn. Corticomuscular coherence with the FD was not different in StimOff vs. HC, but increased with StimOn compared to StimOff $(P=0.039)$. HC showed higher corticomuscular coherence with ED compared to StimOff $(P=0.001)$, and this was unchanged in StimOn. All findings are summarized in Table 2.

Together, at tapping frequency, the corticomuscular coherence findings indicated that synchronization between SM1 area and agonistic FD spinal motor neurons increased with STN-DBS. In StimOff iPD patients had reduced synchronization between SM1 and ED spinal motor neurons compared to HC, but this was unchanged with STN-DBS.
Around $8 \mathrm{~Hz}$, FD activity was similar in HC, StimOff, and StimOn (Fig. 2A, D). ED showed increased activity in StimOff compared to HC $(P=0.039$, Fig. $2 B)$, and this was unchanged with StimOn (Fig. 2E). Intermuscular coherence of antagonistic muscles was stronger in StimOff than in StimOn $(P=0.026$, Fig. $2 \mathrm{~F})$, indicating that higher synchronization between antagonistic spinal motor neurons was reduced by STN-DBS. One may argue that particularly StimOff contained some ULF episodes and this may have confounded our findings. Therefore, we ensured that both FD and ED muscular activity, as well as intermuscular coherence did not differ significantly after removing freezing episodes from the StimOff condition (Section B in the Supplementary material). Cortical activity in StimOff revealed increased activity around $8 \mathrm{~Hz}$ compared to HC $(P=0.002)$ and this activity peak was most pronounced in the bilateral central and parietal regions. This increased cortical activity around $8 \mathrm{~Hz}$ was similar in StimOn with similar topographic distribution (n.s.). Cortical activity was not coherent with FD or ED activity around $8 \mathrm{~Hz}$. All findings are summarized in Table 3.

\subsection{Comparison of 'regular tapping' and 'upper limb freezing' (ULF)}

The verbal fluency task led to more irregular tapping (tapping regularity in StimOn interference $0.67 \pm 0.36$; 'fingertapping only' in StimOn $0.24 \pm 0.15 ; P=0.001$; paired samples $t$-test). Spectral measures were compared between 'regular tapping' and ULF in iPD patients in StimOn interference. Two patients were excluded from this analysis owing to tremor in iPD8 (which occurred only in StimOn interference) and inability to adhere to the interference paradigm in iPD10, respectively. According to criteria one (amplitude decrease $<50 \%$ ) and two (length $>1 \mathrm{~s}$ ), 93 episodes were identified. After applying criterion three $(>3 \mathrm{~Hz}), 21$ episodes were excluded, which might have been related to 'simple movement stopping'. ULF episodes were detected in 9 of 14 iPD patients. The median number of ULF episodes per subject was 7 [5.5 11] (median [IQR]) with a duration of 2.3 [2.0 3.5] seconds. There was no correlation between the duration of ULF (normalized to the whole data length of dual task interference) and the Freezing of Gait Questionnaire item 3 (n.s.).

\subsubsection{Muscular activity and intermuscular coherence during upper limb freezing (ULF)}

The frequency ranges of interest for the statistical analysis of muscular activity were drawn from the grand average plots of muscular activity (Fig. 3). During ULF, both FD and ED showed increased activity around 6 to $9 \mathrm{~Hz}$ (FD, $P=0.021$; ED, $P=0.011$, Fig. 3A, B). During ULF, ED showed reduced activity in the frequency range from 22 to $34 \mathrm{~Hz}(P=0.038$, Fig. $3 \mathrm{~B})$, and no such

Table 2

Motor performance and spectral measures at individual tapping frequency.

\begin{tabular}{|c|c|c|c|c|c|}
\hline & $\mathrm{HC}$ & StimOff & StimOn & StimOff vs. HC & StimOff vs. StimOn \\
\hline \multicolumn{6}{|c|}{ Motor performance of fingertapping } \\
\hline Frequency & $2.16 \pm .93$ & $1.40 \pm .69$ & $1.56 \pm .71$ & 0.009 & 0.04 \\
\hline Regularity & $.16 \pm .07$ & $.31 \pm .16$ & $.24 \pm .15$ & 0.027 & 0.187 \\
\hline \multicolumn{6}{|l|}{ Power } \\
\hline FD & $.14[.08 .17]$ & $.10[.03 .21]$ & $.09[.04 .17]$ & .599 & .569 \\
\hline ED & $.15[.08 .25]$ & $.05[.03 .13]$ & $.05[.03 .14]$ & .012 & .717 \\
\hline \multicolumn{6}{|l|}{ Coherence } \\
\hline Intermuscular coherence & $0.67[0.240 .82]$ & $0.33\left[\begin{array}{ll}0.10 & 0.57\end{array}\right]$ & $0.31\left[\begin{array}{lll}0.04 & 0.60\end{array}\right]$ & 0.056 & 0.959 \\
\hline Corticomuscular FD & $.28[.12 .32]$ & $.11[.03 .21]$ & $.14[.06 .31]$ & .066 & .039 \\
\hline Corticomuscular ED & $.27[.15 .39]$ & $.04[.03 .13]$ & $.09[.05 .25]$ & .001 & .070 \\
\hline
\end{tabular}

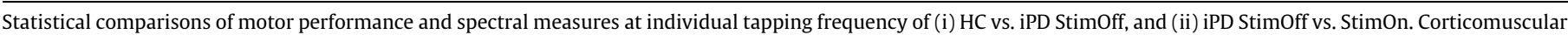

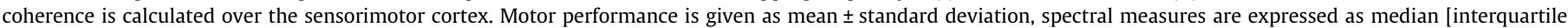

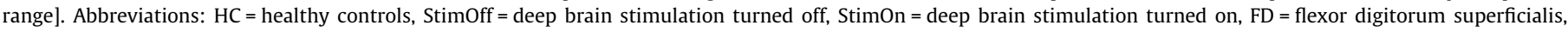
$\mathrm{ED}=$ extensor digitorum communis. 

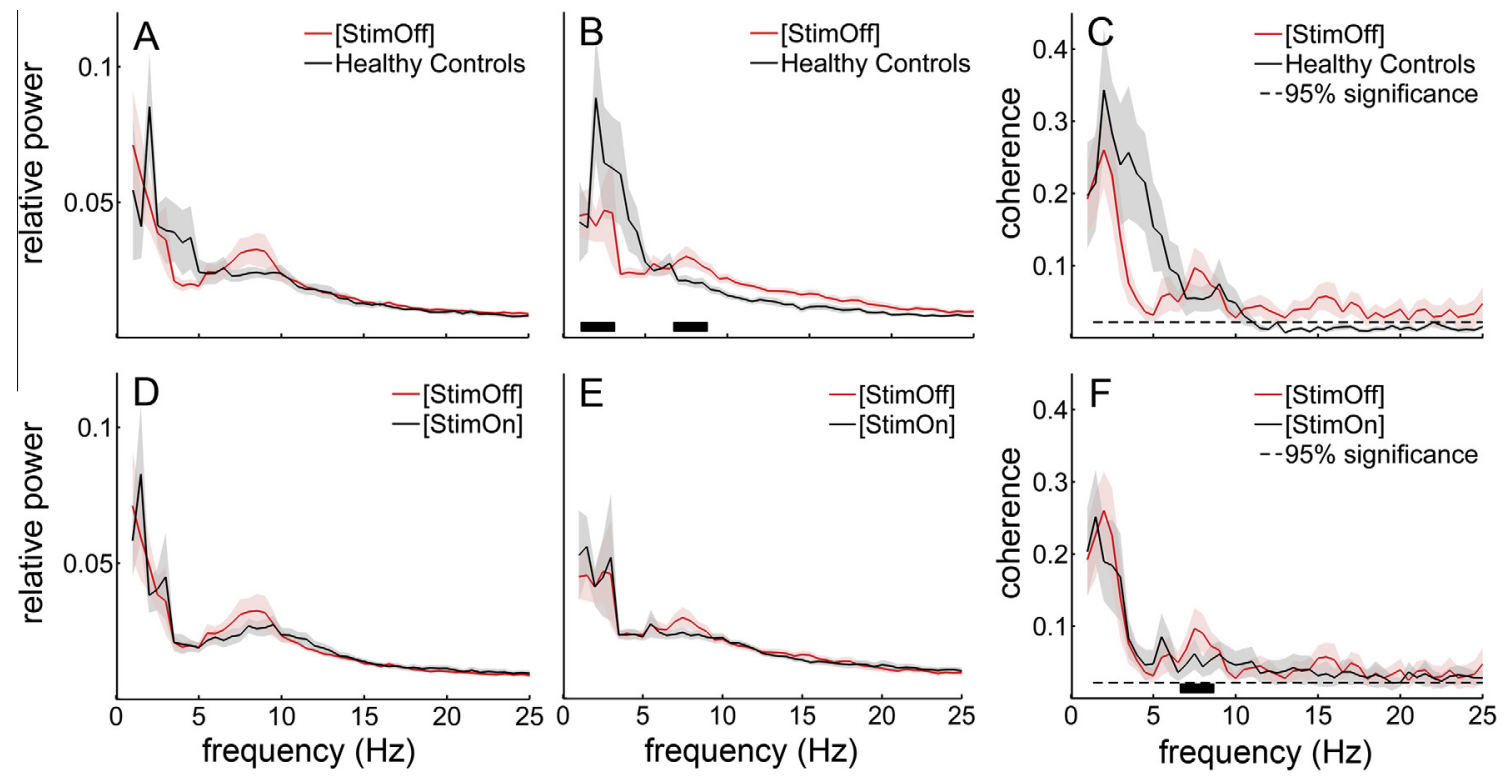

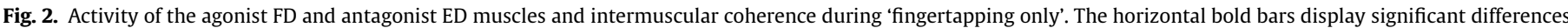

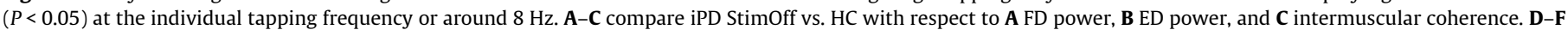

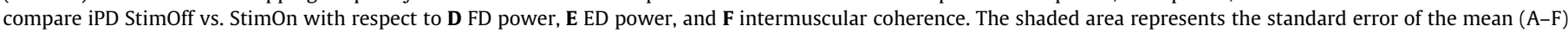
and the dotted line represent the $95 \%$ confidence limit of intermuscular coherence (C and F). $X$-axis: frequency (Hz); $Y$-axis: relative power (a.u.) or coherence.

Table 3

Spectral measures around $8 \mathrm{~Hz}$.

\begin{tabular}{|c|c|c|c|c|c|}
\hline & $\mathrm{HC}$ & StimOff & StimOn & StimOff vs. HC & StimOff vs. StimOn \\
\hline \multicolumn{6}{|l|}{ Power } \\
\hline FD & $.02[.02 .03]$ & $.02[.02 .05]$ & $.02[.02 .03]$ & .539 & .255 \\
\hline ED & $.02[.02 .02]$ & $.03[.02 .04]$ & $.02[.02 .03]$ & .039 & .088 \\
\hline Cortical & $.02[.01 .02]$ & $.03[.02 .03]$ & $.02[.02 .03]$ & .002 & ns. \\
\hline \multicolumn{6}{|l|}{ Coherence } \\
\hline Intermuscular & $.03[.02 .04]$ & $.07[.03 .12]$ & $.03[.01 .07]$ & .219 & .026 \\
\hline Corticomuscular FD & $0.02\left[\begin{array}{ll}0.01 & 0.02\end{array}\right]$ & $0.01\left[\begin{array}{ll}0.01 & 0.01\end{array}\right]$ & $0.01\left[\begin{array}{ll}0.01 & 0.02\end{array}\right]$ & n.a. & n.a. \\
\hline Corticomuscular ED & $0.02\left[\begin{array}{ll}0.01 & 0.02\end{array}\right]$ & $0.01\left[\begin{array}{ll}0.01 & 0.02\end{array}\right]$ & $0.01\left[\begin{array}{ll}0.01 & 0.02\end{array}\right]$ & n.a. & n.a. \\
\hline
\end{tabular}

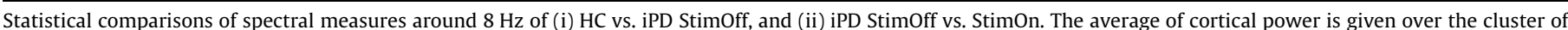

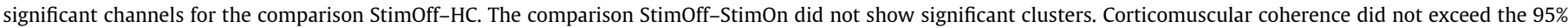

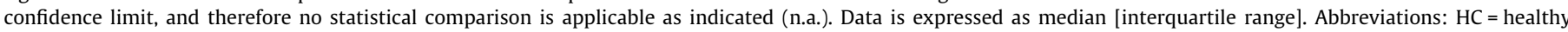
controls, StimOff = deep brain stimulation turned off, StimOn = deep brain stimulation turned on, FD = flexor digitorum superficialis, ED = extensor digitorum communis

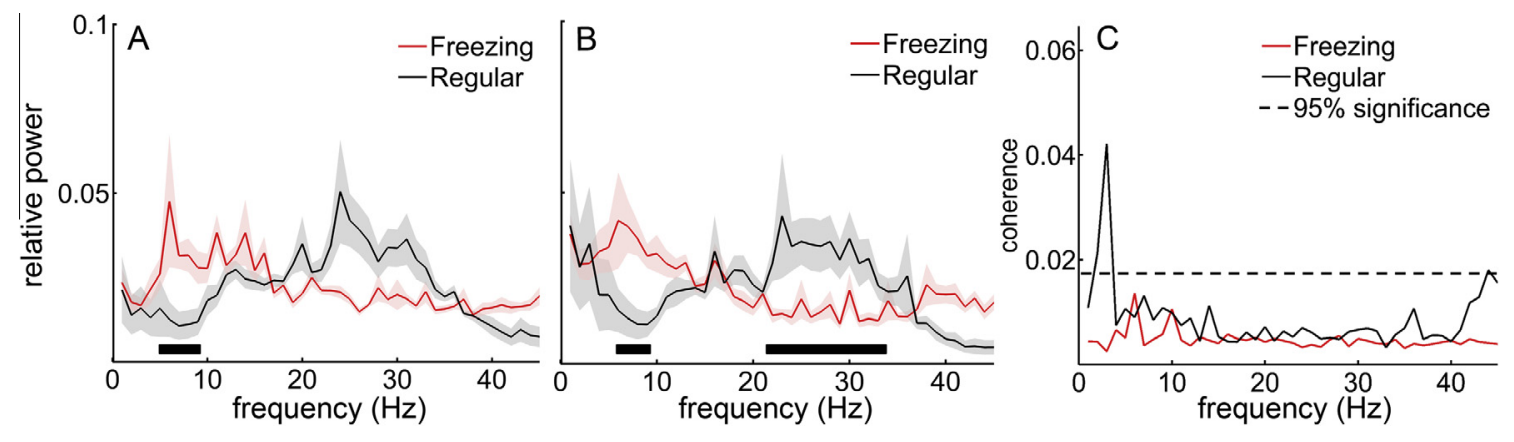

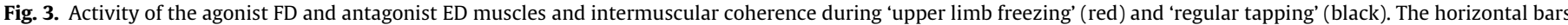

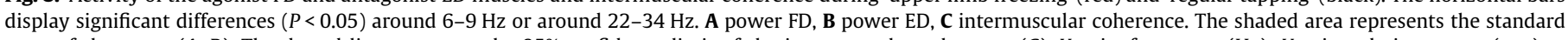

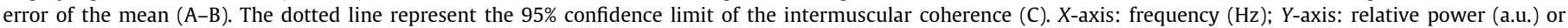
coherence. (For interpretation of the references to colour in this figure legend, the reader is referred to the web version of this article.)

difference was found in FD activity (Fig. 3). There was no significant intermuscular coherence from 6 to $9 \mathrm{~Hz}$ during ULF (Fig. 3C), and the 6 to $9 \mathrm{~Hz}$ muscular activity was not coherent to cortical activity in either FD or ED. All data are summarized in Table 4.

\subsubsection{Cortical activity during upper limb freezing (ULF)}

Cortical activity in the $7-11 \mathrm{~Hz}$ range increased during ULF compared to 'regular tapping' over the left frontal cortex, supplementary motor area, left SM1, and left parietal areas $(P<0.001$, Fig. 4). The time-frequency representations of cortical power from 
Table 4

Spectral measures of muscular activity from 6 to $9 \mathrm{~Hz}$ and from 22 to $34 \mathrm{~Hz}$.

\begin{tabular}{clllllll}
\hline \multirow{2}{*}{ Measure } & $6-9 \mathrm{~Hz}$ & & & $22-34 \mathrm{~Hz}$ \\
\cline { 2 - 3 } & 'Regular' & 'Freezing' & $P$-value & & 'Regular' & 'Freezing' \\
\hline Power & & & & & \\
FD & $.00[.00 .02]$ & $.03[.02 .04]$ & .021 & $.05[.02 .05]$ & $.02[.02 .02]$ & .066 \\
ED & $.00[.00 .03]$ & $.04[.02 .05]$ & .011 & $.03[.02 .05]$ & $.02[.01 .02]$ & .038 \\
\hline
\end{tabular}

Statistical comparisons of spectral measures (i) 6-9 Hz, and (ii) 22-34 Hz in iPD patients. 'Regular tapping' and 'freezing' under dual tasking conditions in StimOn are compared. Data is shown as median [interquartile range]. Abbreviations: FD = flexor digitorum superficialis muscle, $\mathrm{ED}=$ extensor digitorum communis muscle.

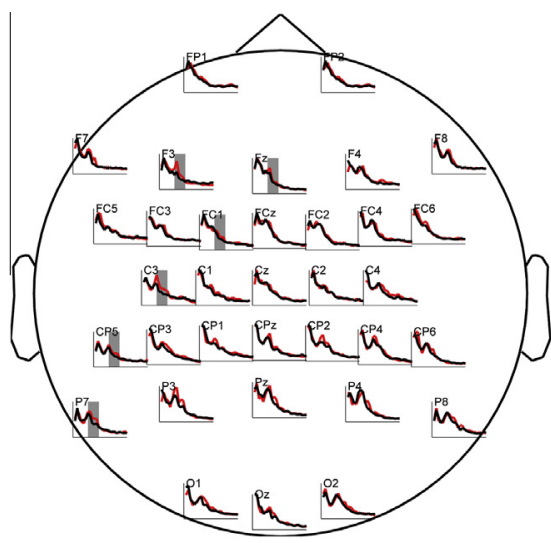

\section{Average of the significant channels}

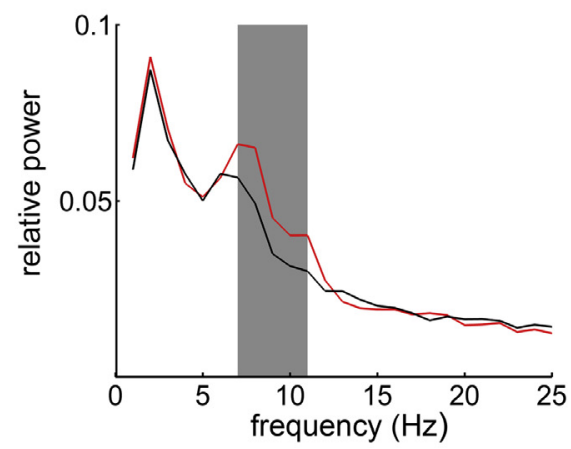

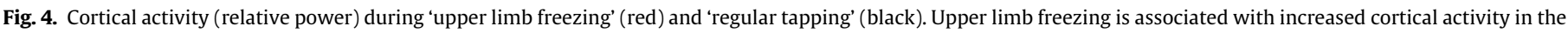

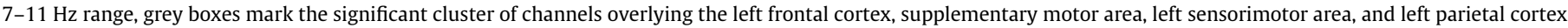

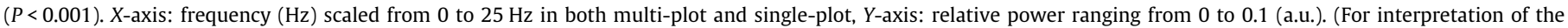
references to colour in this figure legend, the reader is referred to the web version of this article.)

7 to $11 \mathrm{~Hz}$ event-locked to the onset of the ULF episode showed pronounced activation over the left SM1 area $0.7 \mathrm{~s}$ after the last regular finger tap. There was activation spread to the left frontal cortex and to the right parietal area from 0.85 to $1.2 \mathrm{~s}$ (Fig. 5).

\section{Discussion}

During 'fingertapping only', we found that - at individual tapping frequency - STN-DBS increased the corticomuscular coherence with the agonist FD muscle. Additionally, STN-DBS reduced the intermuscular coherence around $8 \mathrm{~Hz}$, whereas STN-DBS did not change the muscular activity. Muscular activity around $8 \mathrm{~Hz}$ was not coherent to cortical activity. ULF compared to 'regular tapping' showed increased muscular activity in the low-frequency range from 6 to $9 \mathrm{~Hz}$, and reduced antagonist activity in the frequency range from 22 to $34 \mathrm{~Hz}$. Cortical activity was increased in the frequency range from 7 to $11 \mathrm{~Hz}$ during ULF, and the time-frequency decompositions of cortical activity showed emergence of left-hemispheric SM1 activity and spread to the left frontal and right parietal areas event-locked to ULF.

\subsection{Subthalamic stimulation reduces low-frequency agonist- antagonist synchronization during repetitive movement}

EMG activity in healthy persons occurs predominantly in the beta band $(15-30 \mathrm{~Hz})$ or Piper rhythm $(35-60 \mathrm{~Hz})($ Brown, 2000). In untreated iPD patients, however, muscle discharge can occur at abnormally low frequencies below $10 \mathrm{~Hz}$, which can be modulated by dopaminergic treatment and STN-DBS (Brown, 1997; Salenius et al., 2002; Weiss et al., 2012). Here, we found pronounced muscular activity around $8 \mathrm{~Hz}$ in iPD patients. Antagonist ED activity was significantly increased in StimOff as compared to HC, but was not modulated by subthalamic stimulation (Fig. 2B, E). Interestingly, however, activity around $8 \mathrm{~Hz}$ showed synchronization between agonists and antagonists in iPD in the StimOff condition, and this was significantly reduced with STN-DBS (Fig. 2F). iPD patients have defective agonist-antagonist activation patterns, which was expressed as lack of inhibition to extensor coupled interneurons during flexion compared to healthy persons (Meunier et al., 2000). This defective 1a-inhibition in iPD seems to be modulated at both cortical (Bertolasi et al., 1998) and subcortical level (Meunier et al., 2000), in particular in the subthalamic nucleus (Raoul et al., 2012). Accordingly, common supraspinal input was described to synchronize the antagonistic spinal motor neuron pools to a common frequency. Previous work demonstrated a loss of beta band synchronization between antagonistic muscles in PD 'off state' which was restored with subthalamic stimulation. This was referred to as facilitation of common corticospinal inputs at the physiological corticomuscular beta frequency (Brown et al., 2001; Marsden et al., 2001). In our study, we found a different and inverse pattern with enhanced agonist-antagonist synchronization in the low-frequency range at around $8 \mathrm{~Hz}$ in the StimOff condition, and attenuation in StimOn. Interestingly, no cortical oscillator was linearly synchronized to this $8 \mathrm{~Hz}$ activity. Mainly, two potential explanations might account for the lack of corticomuscular coherence at $8 \mathrm{~Hz}$. Coupling between cortex and muscle is generally bidirectional with afferent and efferent interactions. A relevant contribution of the afferent component was recently demonstrated in corticokinematic coherence, particularly at frequencies below $10 \mathrm{~Hz}$ (Bourguignon et al., 2015). Accordingly, a jitter in conduction times of afferent and efferent contributions (as is present in non-linear coupling) might result in annihilation of corticomuscular coherence if mathematically expressed as ordinary linear coherence. Alternatively, the lack of corticomuscular coherence at $8 \mathrm{~Hz}$ gives 

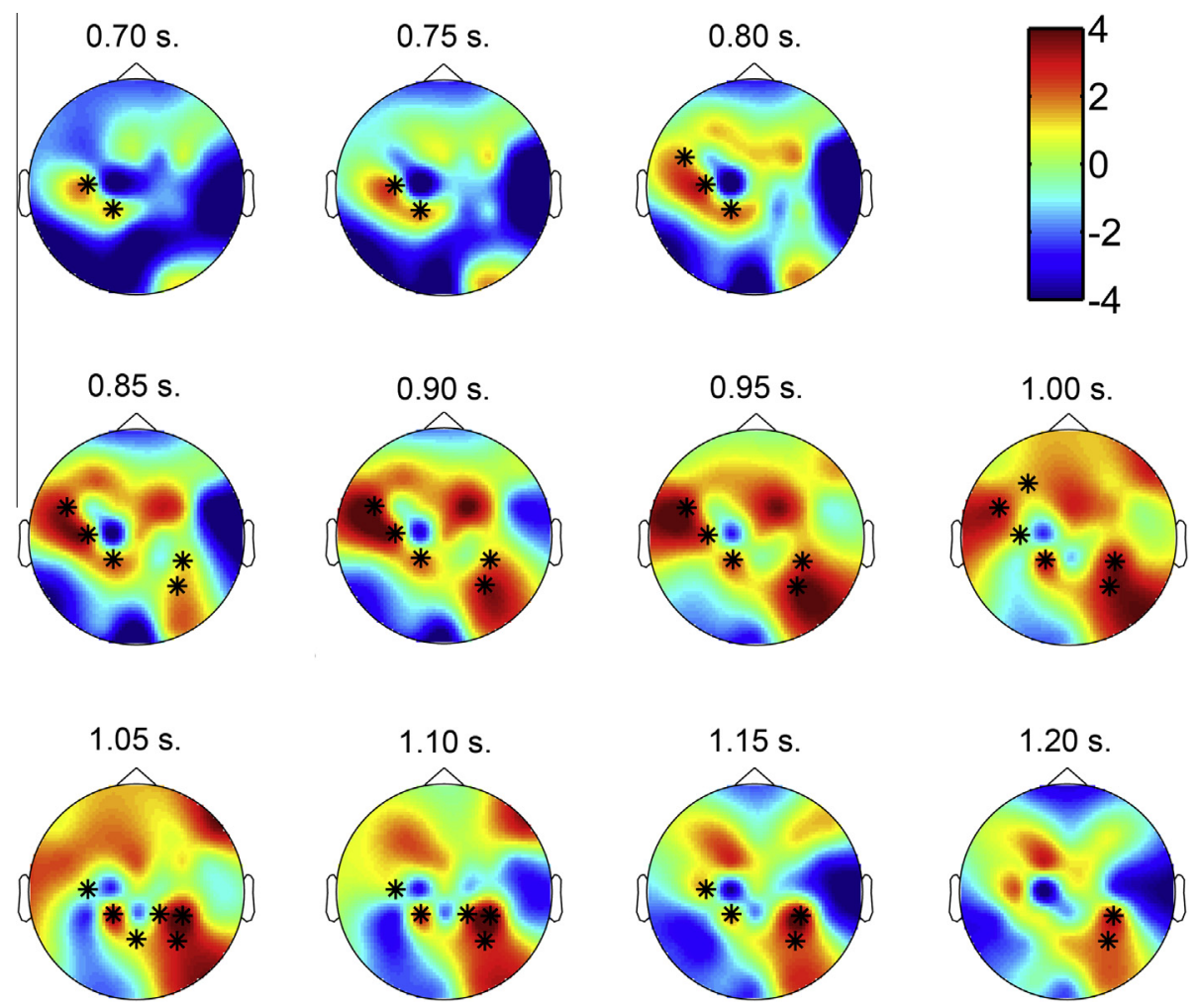

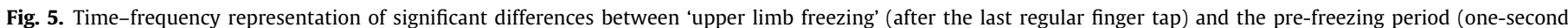

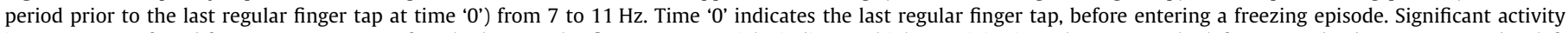

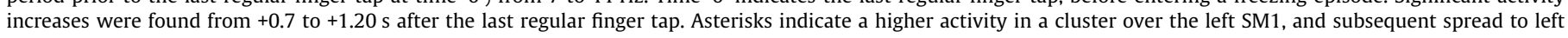
frontal and right parietal cortex $(P=0.012)$. The color bar represents statistical units.

rise to discuss potential motor inputs to the spinal motor neuron pool apart from the corticospinal tract. Motor output from the basal ganglia was demonstrated for the nigro-ponto-reticulospinal pathway and is well known to occur in the startle response. Interestingly, this 'subcortical' descending pathway is characterized by unselective drives to the antagonistic spinal motor neuron pool and can be modulated by both L-Dopa (Delwaide et al., 1993) and subthalamic stimulation (Potter-Nerger et al., 2008). Accordingly, a task-related increase of the 'efferent' subthalamomuscular motor command was identified during isometric finemotor performance compared to rest in iPD (Weiss et al., 2012). The relevance of such pathways for pathological agonist-antagonist synchronization can be addressed in future with combined subthalamic local field potential and EMG recordings.

Another interesting aspect is the long-range synchronization of cortex and muscle at the individual tapping frequency itself. Our data show that cortical synchronization with the spinal agonist motor neurons is increased at tapping frequency with StimOn compared to StimOff and this adds to previous findings on increased corticospinal contributions resulting from subthalamic stimulation (Brown et al., 2001; Kuriakose et al., 2010; Salenius et al., 2002; Weiss et al., 2012). To summarize, we identified two distinct network processes that parallel motor impairment in the PD 'off state': (i) decrease of corticomuscular synchrony at individual tapping frequency, and (ii) emergence of intermuscular synchronization around $8 \mathrm{~Hz}$ without coupling to the cortex.

\subsection{Is transient suppression of cortical motor processing a mechanism of upper limb freezing?}

During ULF, we found enhanced cortical activity from 7 to $11 \mathrm{~Hz}$ in the left SM1, left frontal and left parietal areas (Fig. 4). Similarly, we found in the time-frequency analyses that activity from 7 to $11 \mathrm{~Hz}$ increased during ULF, emerging $0.7 \mathrm{~s}$ after the last regular finger tap. This increased cortical activity showed a temporal-topographic evolution from the left SM1 (i.e., contralateral to the moving hand) to the left frontal and right parietal areas (Fig. 5).

Here, we discuss our findings in the context of previous models on freezing of gait (Nieuwboer and Giladi, 2013): Our paradigm was designed according to the interference hypotheses (inducing a cortical processing conflict by a second cognitive task). Nevertheless, ULF in our study was rather complemented by further mechanisms. In this sense, the so-called 'threshold model' (accumulation of motor deficits until the threshold for freezing is reached) could be considered relevant to our findings, because the tapping was more irregular during StimOn interference compared to StimOn. The 'decoupling model' considers disruption of the pre-planned/automatic movement and emergence of an alternate rhythm in freezing leading to ineffective movement ('block'). A role of the 'decoupling' mechanism to freezing in our study is supported by the findings: the original tapping rhythm and associated corticomuscular coherence at individual tapping frequency collapsed during ULF. Instead, abnormal low-frequency muscle activation from 6 to $9 \mathrm{~Hz}$ was observed as alternate rhythm. Again, this low-frequency muscular activity was not coherent to cortical activity. Similarly, altered cortical activity from 7 to $11 \mathrm{~Hz}$ emerged.

In general, enhanced alpha activity is increasingly recognized as an electrophysiological correlate of cortical motor suppression (Klimesch et al., 2007) and a spatial distribution similar to our study was found during active inhibition of preplanned movement (Sauseng et al., 2012). Interestingly, activity modulations around $10 \mathrm{~Hz}$ are not limited to cortical motor suppression, but also occur at the level of the pedunculopontine nucleus. A transient reduction 
of the pedunculopontine alpha-band power was observed in parallel to freezing of gait (Thevathasan et al., 2012) and coherence modulation between the pedunculopontine nucleus and cortex in the alpha band was identified in phasic wrist movement (Tsang et al., 2010). Whether alpha rhythm modulation might constitute a 'binding mechanism' to balance the contribution of cortico-spinal and nigro-ponto-reticular transmission to the spinal motor neuron might be addressed in future with combined electrophysiological recordings from the midbrain and brainstem nuclei together with MEG/EEG and EMG. Together, we consider the abnormal increase in cortical activity in the alpha frequency range in ULF most likely as an inhibitory mechanism in the active sensorimotor cortex. The disruption of corticomuscular synchronization in ULF at the freezing rhythm itself further argues into this direction and attracts interest on alternate (presumably subcortical) drivers.

\subsection{Methodological considerations}

Several methodological considerations have to be taken into account when interpreting our findings on ULF. We aimed to operationalize detection and data segmentation of the ULF episodes. Hitherto, there is no 'gold standard' in identifying ULF episodes and in defining an accurate onset. This is inherent to research on freezing phenomena that develop with variable time delay after a regular movement and may be preceded by a short period of movement slowing and amplitude decrease before "trembling-in-place-like-freezing' emerges (Nutt et al., 2011). Therefore, the most reproducible and reliable way to standardize the segmentation of our time-frequency analyses was to time-lock to the last regular finger tap before ULF emerged. We aimed to stabilize this natural variability by combining several earlier constraints on freezing pathophysiology. By selecting only ULF episodes with frequency $>3 \mathrm{~Hz}$, we assumed that freezing behavior has a generic pathophysiological mechanism, because this criterion was demonstrated as common spatiotemporal characteristic in ULF (Vercruysse et al., 2012) and freezing of gait (Delval et al., 2010). Following this constraint, we rejected 21 out of 93 episodes ULF episodes in StimOn interference that might have related to 'simple movement stopping'.

Moreover, ULF episodes were only selected during StimOn interference, although ULF episodes also occurred in StimOff and to a lesser extent in StimOn. However, the concatenation of freezing episodes of all therapeutic conditions would have yielded less consistent results from a clinical standpoint. Furthermore, we primarily intended to capture ULF episodes resistant to subthalamic neurostimulation. On the other hand, we considered that during dual task interference ULF is provoked due to a cortical processing conflict and limited attentional resources (Bloem et al., 2001; Yogev et al., 2005). This includes that the patients transiently might 'forget to tap', i.e., stop their attempt to overcome the motor suppression. However, we found increased low-frequency motor output of the antagonistic muscles during the ULF episodes. This is not compatible with 'motor inactivity' as could be expected if patients shifted their attention away from the motor task and towards the verbal fluency task.

We further selected for patients with akinetic-rigid symptom dominance, and therefore the results should not be generalized to the whole iPD population. Nevertheless, this selection criterion was necessary to prevent a potential and confounding influence of tremor on our findings. Accordingly, we inspected each individual EMG data set for tremor and, consecutively, one patient had to be excluded when tremor solely occurred during StimOn interference. Generally however, the ULF phenomenon in this study and in rich previous work is separable from action tremor despite of its 'trembling-like' nature for several major reasons that were also conceived in our study (Barbe et al., 2014; Nieuwboer et al.,
2009; Nutt et al., 2011): during ULF the power spectrum of the biomechanical signal traces did not show a distinct peak at tremor frequency (unlike the excluded measurement of iPD8, who showed a tremor peak). Characteristic for ULF, the power spectrum of the biomechanical signal showed broader low-frequency activity (exemplary data provided as Supplementary material, Section A). This was referred to as 'unevenness of the energy spectrum' (Nutt et al., 2011) and considered as typical feature of freezing phenomena, and therefore constitutes a motor phenomenon different from PD resting or action tremor that generally occurs at a constant and circumscribed frequency (Nutt et al., 2011). Typically, PD tremor also occurs with significant corticomuscular coherence at single and double tremor frequency, which was not observed in our spectral analysis of ULF (Hirschmann et al., 2013; Timmermann et al., 2003). Finally, it should be recognized that the mechanisms on ULF identified here should only carefully be translated to freezing of gait (Barbe et al., 2014). Nevertheless, our work generates important motor network hypotheses for future research on freezing of gait pathophysiology.

\section{Conclusion}

In conclusion, we propose that subthalamic stimulation strengthens the synchronization of cortical and agonist muscle activity at individual tapping frequency, and reduces increased low-frequency agonist-antagonist synchronization around $8 \mathrm{~Hz}$. Upper limb freezing is reflected by muscular low-frequency activation around 6 to $9 \mathrm{~Hz}$ and cortical overactivity in the frequency range from 7 to 11 . Together, our findings bring to light several network hypotheses that are valuable for future research to address freezing of gait, an unmet therapeutic need in Parkinson's disease.

\section{Funding}

This work was supported by a research grant of St. Jude Medical Inc.

\section{Acknowledgements}

Marlieke Scholten, Rosa Klotz, Christian Plewnia, Bastiaan R. Bloem, Christoph Braun, Ulf Ziemann, and Rathinaswamy B. Govindan report no competing financial interest.

Tobias Wächter received financial support for research from and conducted commissioned research for Medtronic.

Carina Mielke was supported by a research grant of St. Jude Medical.

Alireza Gharabaghi is supported by a grant from Medtronic.

Rejko Krüger has received speaker's honoraria and/or travel grants from St. Jude Medical, and Medtronic.

Daniel Weiss received research funding, speaker's honoraria, and a travel grant from Medtronic and a research grant from the German Research Council (DFG, WE5375/1-1).

\section{Appendix A. Supplementary data}

Supplementary data associated with this article can be found, in the online version, at http://dx.doi.org/10.1016/j.clinph.2015.02. 012.

\section{References}

Barbe MT, Amarell M, Snijders AH, Florin E, Quatuor EL, Schonau E, et al. Gait and upper limb variability in Parkinson's disease patients with and without freezing of gait. J Neurol 2014;261:330-42. 
Bertolasi L, Priori A, Tinazzi M, Bertasi V, Rothwell JC. Inhibitory action of forearm flexor muscle afferents on corticospinal outputs to antagonist muscles in humans. J Physiol 1998;511:947-56.

Bloem BR, Grimbergen YA, van Dijk JG, Munneke M. The, "posture second” strategy: a review of wrong priorities in Parkinson's disease. J Neurol Sci 2006;248:196-204.

Bloem BR, Valkenburg VV, Slabbekoorn M, van Dijk JG. The multiple tasks test. Strategies in Parkinson's disease. Exp Brain Res 2001;137:478-86.

Bourguignon M, Piitulainen H, De Tiege X, Jousmaki V, Hari R. Corticokinematic coherence mainly reflects movement-induced proprioceptive feedback. Neuroimage 2015;106:382-90.

Brown P. Muscle sounds in Parkinson's disease. Lancet 1997;349:533-5.

Brown P. Cortical drives to human muscle: the Piper and related rhythms. Prog Neurobiol 2000;60:97-108.

Brown P, Marsden J, Defebvre L, Cassim F, Mazzone P, Oliviero A, et al. Intermuscular coherence in Parkinson's disease: relationship to bradykinesia. Neuroreport 2001;12:2577-81.

Camicioli R, Oken BS, Sexton G, Kaye JA, Nutt JG. Verbal fluency task affects gait in Parkinson's disease with motor freezing. J Geriatr Psychiatry Neurol $1998 ; 11: 181-5$

Cooper SE, McIntyre CC, Fernandez HH, Vitek JL. Association of deep brain stimulation washout effects with Parkinson disease duration. JAMA Neurology 2013;70:95-9.

Delorme A, Makeig S. EEGLAB: an open source toolbox for analysis of single-trial EEG dynamics including independent component analysis. J Neurosci Methods 2004:134:9-21.

Delval A, Snijders AH, Weerdesteyn V, Duysens JE, Defebvre L, Giladi N, et al. Objective detection of subtle freezing of gait episodes in Parkinson's disease. Mov Disord 2010;25:1684-93.

Delwaide PJ, Pepin JL, Maertens de Noordhout A. The audiospinal reaction in Parkinsonian patients reflects functional changes in reticular nuclei. Ann Neurol 1993;33:63-9.

Deuschl G, Schade-Brittinger C, Krack P, Volkmann J, Schafer H, Botzel K, et al. A randomized trial of deep-brain stimulation for Parkinson's disease. N Engl J Med 2006;355:896-908.

Devos D, Labyt E, Derambure P, Bourriez JL, Cassim F, Reyns N, et al. Subthalamic nucleus stimulation modulates motor cortex oscillatory activity in Parkinson's disease. Brain 2004;127:408-19.

Farmer SF, Bremner FD, Halliday DM, Rosenberg JR, Stephens JA. The frequency content of common synaptic inputs to motoneurones studied during voluntary isometric contraction in man. J Physiol 1993;470:127-55.

Fling BW, Cohen RG, Mancini M, Nutt JG, Fair DA, Horak FB. Asymmetric pedunculopontine network connectivity in parkinsonian patients with freezing of gait. Brain 2013;136:2405-18.

Folstein MF, Folstein SE, McHugh PR. "Mini-mental state". A practical method for grading the cognitive state of patients for the clinician. J Psychiatr Res 1975;12:189-98.

Giladi N, Hausdorff JM. The role of mental function in the pathogenesis of freezing of gait in Parkinson's disease. J Neurol Sci 2006;248:173-6.

Giladi N, Shabtai H, Simon ES, Biran S, Tal J, Korczyn AD. Construction of freezing of gait questionnaire for patients with Parkinsonism. Parkinsonism Relat Disord 2000;6:165-70

Herman T, Giladi N, Hausdorff JM. Neuroimaging as a window into gait disturbances and freezing of gait in patients with Parkinson's disease. Curr Neurol Neurosci Rep 2013;13:411.

Hirschmann J, Hartmann CJ, Butz M, Hoogenboom N, Ozkurt TE, Elben S, et al. A direct relationship between oscillatory subthalamic nucleus-cortex coupling and rest tremor in Parkinson's disease. Brain 2013;136:3659-70.

Hjorth B. Source derivation simplifies topographical EEG interpretation. Am J EEG Technol 1980;20:121-32.

Jessell TM, Surmeli G, Kelly JS. Motor neurons and the sense of place. Neuron 2011;72:419-24.

Kilner JM, Baker SN, Salenius S, Hari R, Lemon RN. Human cortical muscle coherence is directly related to specific motor parameters. J Neurosci $2000 ; 20: 8838-45$.

Klimesch W, Sauseng P, Hanslmayr S. EEG alpha oscillations: the inhibition-timing hypothesis. Brain Res Rev 2007;53:63-88.

Krack P, Batir A, Van Blercom N, Chabardes S, Fraix V, Ardouin C, et al. Five-year follow-up of bilateral stimulation of the subthalamic nucleus in advanced Parkinson's disease. N Engl J Med 2003;349:1925-34.

Kuriakose R, Saha U, Castillo G, Udupa K, Ni Z, Gunraj C, et al. The nature and time course of cortical activation following subthalamic stimulation in Parkinson's disease. Cereb Cortex 2010;20:1926-36.

Maris E, Oostenveld R. Nonparametric statistical testing of EEG- and MEG-data. J Neurosci Methods 2007;164:177-90.

Marsden J, Limousin-Dowsey P, Fraix V, Pollak P, Odin P, Brown P. Intermuscular coherence in Parkinson's disease: effects of subthalamic nucleus stimulation. Neuroreport 2001;12:1113-7.

Meunier S, Pol S, Houeto JL, Vidailhet M. Abnormal reciprocal inhibition between antagonist muscles in Parkinson's disease. Brain 2000;123:1017-26.

Mima T, Hallett M. Corticomuscular coherence: a review. J Clin Neurophysiol 1999;16:501-11.

Nieuwboer A, Giladi N. Characterizing freezing of gait in Parkinson's disease: models of an episodic phenomenon. Mov Disord 2013;28:1509-19.

Nieuwboer A, Vercruysse S, Feys P, Levin O, Spildooren J, Swinnen S. Upper limb movement interruptions are correlated to freezing of gait in Parkinson's disease. Eur J Neurosci 2009;29:1422-30.
Nutt JG, Bloem BR, Giladi N, Hallett M, Horak FB, Nieuwboer A. Freezing of gait: moving forward on a mysterious clinical phenomenon. Lancet Neurol 2011; 10:734-44

O'Shea S, Morris ME, Iansek R. Dual task interference during gait in people with Parkinson disease: effects of motor versus cognitive secondary tasks. Phys Ther 2002;82:888-97.

Oostenveld R, Fries P, Maris E, Schoffelen JM. FieldTrip: Open source software for advanced analysis of MEG, EEG, and invasive electrophysiological data. Comput Intell Neurosci 2011:2011:156869.

Potter-Nerger M, Ilic TV, Siebner HR, Deuschl G, Volkmann J. Subthalamic nucleus stimulation restores corticospinal facilitation in Parkinson's disease. Mov Disord 2008;23:2210-5.

Rahman S, Griffin HJ, Quinn NP, Jahanshahi M. The factors that induce or overcome freezing of gait in Parkinson's disease. Behav Neurol 2008;19:127-36.

Raoul S, Roualdes V, Deligny C, Leduc D, Lamy JC, Lackmy-Vallee A, et al. Subthalamic nucleus stimulation reverses spinal motoneuron activity in parkinsonian patients. Brain 2012;135:139-47.

Roach BJ, Mathalon DH. Event-related EEG time-frequency analysis: an overview of measures and an analysis of early gamma band phase locking in schizophrenia. Schizophr Bull 2008;34:907-26.

Rosenberg JR, Amjad AM, Breeze P, Brillinger DR, Halliday DM. The Fourier approach to the identification of functional coupling between neuronal spike trains. Prog Biophys Mol Biol 1989;53:1-31.

Salenius S, Avikainen S, Kaakkola S, Hari R, Brown P. Defective cortical drive to muscle in Parkinson's disease and its improvement with levodopa. Brain 2002:125:491-500.

Sauseng P, Gerloff C, Hummel FC. Two brakes are better than one: The neural bases of inhibitory control of motor memory traces. Neuroimage. 2012;65C:52-8.

Schneider TR, Debener S, Oostenveld R, Engel AK. Enhanced EEG gamma-band activity reflects multisensory semantic matching in visual-to-auditory object priming. Neuroimage 2008;42:1244-54.

Schuepbach WM, Rau J, Knudsen K, Volkmann J, Krack P, Timmermann L, et al. Neurostimulation for Parkinson's disease with early motor complications. N Engl J Med 2013;368:610-22.

Shine JM, Handojoseno AM, Nguyen TN, Tran Y, Naismith SL, Nguyen H, et al Abnormal patterns of theta frequency oscillations during the temporal evolution of freezing of gait in Parkinson's disease. Clin Neurophysiol 2014;125:569-76.

Shine JM, Matar E, Ward PB, Bolitho SJ, Pearson M, Naismith SL, et al. Differential neural activation patterns in patients with Parkinson's disease and freezing of gait in response to concurrent cognitive and motor load. PLoS One 2013a;8:e52602.

Shine JM, Moustafa AA, Matar E, Frank MJ, Lewis SJ. The role of frontostriatal impairment in freezing of gait in Parkinson's disease. Front Syst Neurosci 2013b;7:61.

Silberstein P, Pogosyan A, Kuhn AA, Hotton G, Tisch S, Kupsch A, et al. Corticocortical coupling in Parkinson's disease and its modulation by therapy. Brain 2005;128:1277-91.

Spildooren J, Vercruysse S, Desloovere K, Vandenberghe W, Kerckhofs E, Nieuwboer A. Freezing of gait in Parkinson's disease: the impact of dual-tasking and turning. Mov Disord 2010;25:2563-70.

Thevathasan W, Pogosyan A, Hyam JA, Jenkinson N, Foltynie T, Limousin P, et al Alpha oscillations in the pedunculopontine nucleus correlate with gait performance in parkinsonism. Brain 2012;135:148-60.

Timmermann L, Gross J, Dirks M, Volkmann J, Freund H, Schnitzler A. The cerebral oscillatory network of parkinsonian resting tremor. Brain 2003;126:199-212.

Tsang EW, Hamani C, Moro E, Mazzella F, Poon YY, Lozano AM, et al. Involvement of the human pedunculopontine nucleus region in voluntary movements. Neurology 2010;75:950-9.

Vercruysse S, Spildooren J, Heremans E, Vandenbossche J, Wenderoth N, Swinnen SP, et al. Abnormalities and cue dependence of rhythmical upper-limb movements in Parkinson patients with freezing of gait. Neurorehabil Neural Repair 2012;26:636-45.

Vercruysse S, Spildooren J, Heremans E, Wenderoth N, Swinnen SP, Vandenberghe W, et al. The neural correlates of upper limb motor blocks in Parkinson's disease and their relation to freezing of gait. Cereb Cortex 2013;24:3154-66.

Vercruysse S, Vandenberghe W, Munks L, Nuttin B, Devos H, Nieuwboer A. Effects of deep brain stimulation of the subthalamic nucleus on freezing of gait in Parkinson's disease: a prospective controlled study. J Neurol Neurosurg Psychiatry 2014;5:871-7.

Ward NJ, Farmer SF, Berthouze L, Halliday DM. Rectification of EMG in low force contractions improves detection of motor unit coherence in the beta-frequency band. J Neurophysiol 2013;110:1744-50.

Weiss D, Breit S, Hoppe J, Hauser AK, Freudenstein D, Kruger R, et al. Subthalamic nucleus stimulation restores the efferent cortical drive to muscle in parallel to functional motor improvement. Eur J Neurosci 2012;35:896-908.

Weiss D, Klotz R, Rathinaswamy RB, Scholten M, Naros G, Ramos-Murguialday A, et al. Subthalamic stimulation modulates cortical motor network activity and synchronization in Parkinson's Disease. Brain 2015. http://dx.doi.org/10.1093/ brain/awu380.

Weiss D, Walach M, Meisner C, Fritz M, Scholten M, Breit S, et al. Nigral stimulation for resistant axial motor impairment in Parkinson's disease? A randomized controlled trial. Brain 2013;136:2098-108.

Yogev G, Giladi N, Peretz C, Springer S, Simon ES, Hausdorff JM. Dual tasking, gait rhythmicity, and Parkinson's disease: which aspects of gait are attention demanding? Eur J Neurosci 2005;22:1248-56. 